\title{
POWER SPECTRA FOR GALAXY SHAPE CORRELATIONS
}

\author{
JONATHAN MACKEY \\ Harvard-Smithsonian Center for Astrophysics, 60 Garden St., \\ Cambridge, MA 02138, USA \\ E-mail: jmackey@cfa.harvard.edu
}

\begin{abstract}
It has recently been argued that the observed ellipticities of galaxies may be determined at least in part by the primordial tidal gravitational field in which the galaxy formed. Long-range correlations in the tidal field could thus lead to an ellipticity-ellipticity correlation for widely separated galaxies. I present results of a calculation of the angular power spectrum of intrinsic galaxy shape correlations using a new model relating ellipticity to angular momentum. I show that for low redshift galaxy surveys, the model predicts that intrinsic correlations will dominate correlations induced by weak lensing, in good agreement with previous theoretical work and observations. The model also produces ' $E$-mode' correlations enhanced by a factor of 3.5 over ' $B$-modes' on small scales, making it harder to disentangle intrinsic correlations from weak lensing.
\end{abstract}

\section{Introduction}

The stydy of galaxy alignments has a rich history, as documented by Djorgovskit, although with mixed results (e.g. Cabanela \& Aldering E). Recently, Lee \& Pen 9 investigated a relationship between galaxy spins and the underlying gravitational potential field with a view to reconstructing this potential. It was quickly realised that spatial correlations in the gravitational potential could induce correlations in the spins of nearby galaxies. This is interesting in its own right, but is also a potential contaminant of field-surveys for weak gravitational lensing by large scale structure.

Weak lensing shear, the coherent distortion of galaxyimages on the sky induced by density perturbations along the line of sight 11 has now been detected by several different groups 13 . Following standard practice, all of these authors assume that all of their observed correlation in the ellipticities of galaxies comes from weak lensing. Intrinsic shape correlations, if present, should be considered when interpreting results from these field-lensing surveys.

Several authors have investigated these intrinsic galaxy shape correlations

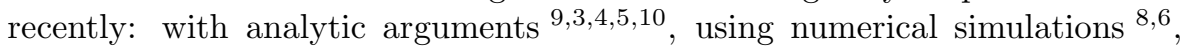
and with observations 12 . The calculation and results presented below can be found in more detail in Mackey, White \& Kamionkowski 10. Our falculation builds on ideas contained in Catelan, Kamionkowski \& Blandford 3 , and uses some similar physical assumptions to Crittenden et al. . 


\section{Ellipticity Model and Power Spectra Calculation}

Here I briefly discuss the model and calculation. Using the same formalism as in weak lensing analyses, I describe the intrinsic ellipticity of galaxies in terms of the spin-2 (complex) ellipticity: $\epsilon=|\epsilon| e^{2 i \phi}=\gamma_{1}+i \gamma_{2}$. I assume the components $\gamma_{i}$ of the ellipticity are determined by a galaxy's angular momentum. This makes sense in that a rotating system will become flattened perpendicular to the angular momentum vector, and will be more flattened for systems with higher angular momentum. In this case, as argued by Catelan et al. $\mathrm{B}$, the intrinsic ellipticity is given by

$$
\gamma_{1}=f\left(L, L_{z}\right)\left(L_{x}^{2}-L_{y}^{2}\right) \text { and } \gamma_{2}=2 f\left(L, L_{z}\right) L_{x} L_{y},
$$

where the sky is the $x$ - $y$ plane, and where $f\left(L, L_{z}\right)$ is an unknown function which determines how ellipticity scales with $L$. I take $f\left(L, L_{z}\right)$ to be a constant, $C$, whose value must be fitted empirically to the observed rms ellipticity of galaxies. This means that $|\epsilon| \propto L^{2}$.

Galaxies acquire angular momentum during formation by tidal torques. It can be shown 14 that the angular momentum acquired to first order in the gravitational potential $\Phi(\mathbf{x})$, is $L_{i} \propto \epsilon_{i j k} I_{k l} \partial_{l} \partial_{j} \Phi(\mathbf{x})$, where $I_{i j}$ is the protogalaxy's inertia tensor.

The ellipticity components $\gamma_{i}$ can thus be calculated in terms of the gravitational potential. I then decompose the spin-2 ellipticity field into scalar (electric-type $E$-mode) and pseudo-scalar (magnetic-type $B$-mode) fields in Fourier space. This enables the construction of $3 \mathrm{D}$ power spectra for the ellipticities, which are convolutions over the density power spectrum. Finally, the Limber approximation is used to obtain the predicted angular power spectrum for different source galaxy distributions.

\section{Results and Discussion}

To demonstrate the results I use a low and a high redshift source distribution (with mean source redshifts $\left\langle z_{\text {src }}\right\rangle=0.1$ and 1.0). The ellipticity-ellipticity angular power spectra obtained are shown in Fig. 1, along with the corresponding weak lensing prediction.

The most obvious feature in Fig. 1 is that for a low redshift survey, intrinsic correlations are expected to dominate over weak lensing signal, while with $\left\langle z_{\text {src }}\right\rangle=1.0$, they are a very small contaminant to weak lensing measurements. The reasons for this result are twofold: the lensing signal is proportional to the projected density which increases with survey depth, while the intrinsic signal is increasingly washed out by projection effects for deeper surveys. The predicted amplitudes are in good agreement with the calculations of Crittenden 

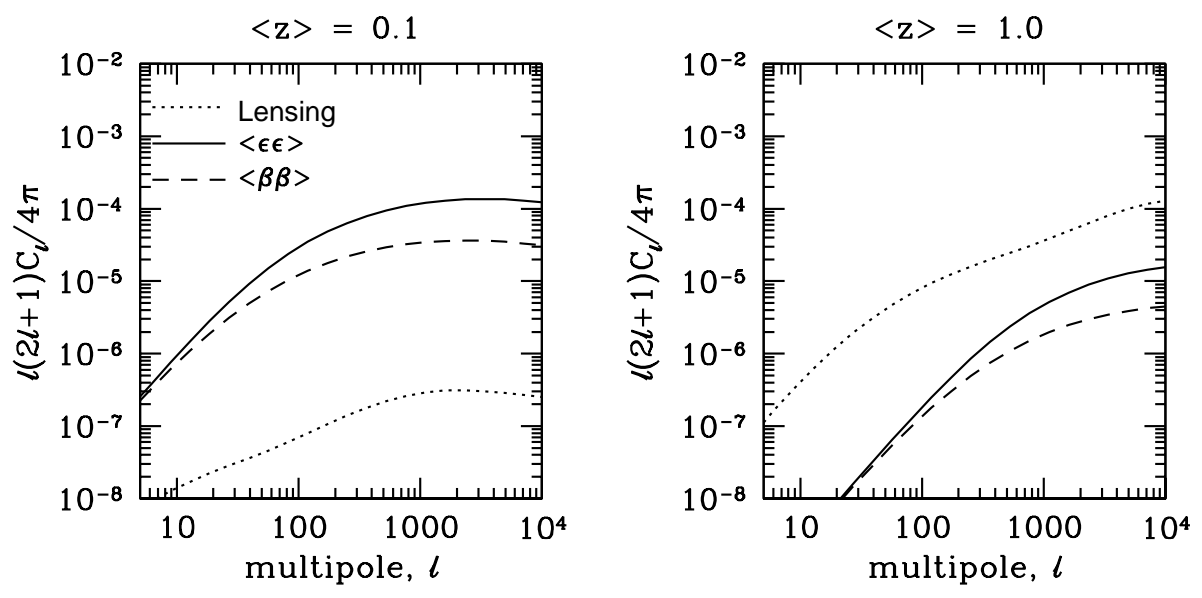

Figure 1: The angular power spectra of intrinsic shape correlations as predicted by this model. The solid line is the $E E$, and the dashed line the $B B$ intrinsic power spectrum. The $E B$ cross power spectrum is parity violating, so it is identically zero. For comparison, the dotted line is the predicted weak lensing signal. The left panel is for a low redshift source distribution with mean $\left\langle z_{\text {src }}\right\rangle=0.1$, and the right panel a high redshift distribution with mean $\left\langle z_{\mathrm{src}}\right\rangle=1.0$. For reference, $\ell \sim 200$ corresponds to an angular scale of $\theta \sim 1^{\circ}$.

et al , and comparable to the observational results of Brown et a 1 i over angular scales from $1 \Omega^{\prime}-100^{\prime}$. They are also comparable to findings from numerical simulations 6 6.

The shape of the angular power spectrum reflects the shape of the underlying density power spectrum $\Delta_{m}^{2}(k)$, going roughly flat on small scales. On large scales the log-slope is 2 , consistent with shot noise. The shapes of the intrinsic and lensing power spectra are quite similar, making it difficult to use this to separate the two components.

A potentially better discriminant is given by the relative levels of $E$ - and $B$ -

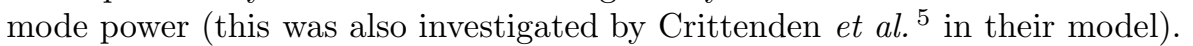
Weak lensing shear has no handedness and can therefore produce only $E$ modes. Fig. 1 shows that the intrinsic power spectra have equal $E$ - and $B$ mode power on large scales, but $C_{\ell}^{\epsilon \epsilon} / C_{\ell}^{\beta \beta} \simeq 3.5$ on small scales. This has two important implications for weak lensing. First, a detection of $B$-mode power would indicate that intrinsic correlations are present. Second, intrinsic correlations can still hide in a low signal-to-noise measurement of $E$-mode only power (but only up to a factor of 3.5), because of this $E$-mode enhancement on small scales. Thus, some caution should be used in estimating noise and contamination of weak lensing measurements solely from the level of $B$-mode 
power.

The $E$-mode enhancement can be understood intuitively by noting that isolated point masses can generate only $E$-modes. On smaller scales the density field can be increasingly described in terms of distinct objects, giving a mostly $E$-mode signal. $B$-modes arise because the ellipticities $\gamma_{i}$ are convolutions over the Fourier modes of the potential. It was found that large scale perturbations on the small scale potential field produce the $B$-modes.

The small scale $E$-mode enhancement is a distinctive feature of this model, and could be tested with current observational data. It is qualitatively different from that of Crittenden et al. 5, who found that $E$-and $B$-modes are the same on small scales and different on large scales. The halo shapes ellipticity model of Catelan et al. 3 is also different in that it produces only $E$-modes. Thus, the $E$ - $B$ decomposition is potentially a very good way to observationally

distinguish between the different models of intrinsic correlations. Determining the correct model may give us new insight into galaxy formation and evolution processes, so it is important to do so.

\section{Acknowledgments}

I thank Martin White and Marc Kamionkowski for collaboration and advice on this work. Thanks especially to MW for many hours of discussion and lots of good ideas and guidance.

\section{References}

[1] Brown, M. L. et al., 2000, astro-ph/0009499.

[2] Cabanela J. E. \& Aldering G., 1998, AJ, 116, 1094

[3] Catelan P., Kamionkowski M., Blandford R.D., 2000, MNRAS, 320, 7.

[4] Crittenden, R. G. et al., 2000, astro-ph/0009052.

[5] Crittenden, R. G. et al., 2000, astro-ph/0012336.

[6] Croft R. \& Metzler C., 2000, ApJ, 545, 561.

[7] Djorgovski S., 1987, in Nearly Normal Galaxies, (Springer, New York), p. 227.

[8] Heavens A., Refregier A., Heymans C., 2000, MNRAS, 319, 649.

[9] Lee J., \& Pen U.-L., 2000, ApJ, 532, L5.

[10] Mackey, J., White, M., Kamionkowski, M., astro-ph/0106364

[11] Mellier, Y., 1999, ARA\&A, 37, 127 (and references therein).

[12] Pen U.-L., Lee J., Seljak U., 2000, ApJ, 543, L107.

[13] Rhodes J., Refregier A., Groth E. J., 2001, ApJ, 552, L85 (and references therein).

[14] White S. D. M., 1984, ApJ, 286, 38. 